\title{
Effect of Preemergence Herbicide on Wildflower Establishment
}

\author{
K.S. Erusha ${ }^{1}$ C. Fricker ${ }^{2}$, R.C. Shearman ${ }^{3}$, and D.H. Steinegger ${ }^{4}$ \\ University of Nebraska, Lincoln, NE 68583-0724
}

This study was initiated to evaluate wildflower establishment using a preemergence herbicide. Fifty species of wildflowers (Table 1) were established on a Sharpsburg silty-clay loam (Typic Argiudoll), with a $6.9 \mathrm{pH}$, near Mead, Neb. Fortyseven (Table 1) wildflowers were established near Hubbard, Ore., on a Willamette sandy loam (Pachic Ultic Argixeroll) with a $6.6 \mathrm{pH}$. Both studies used a split-plot design, with herbicide treatment as main plots and wildflowers as subplots. Each treatment consisted of two replicates.

Herbicide treatments included an untreated control and a combination of S-ethyl dipropylthiocarbamate (EPTC) at $2.3 \mathrm{~kg} \cdot \mathrm{ha}^{-1}$ and $\alpha, \alpha, \alpha$-trifluoro-2,6-dinitro-N,N-dipropyl-ptoluidine (trifluralin) at $0.6 \mathrm{~kg} \cdot \mathrm{ha}^{-1}$. Applications were made with a boom-sprayer, and treatments were incorporated with a Lily Roterra (Lely Industries, N.V. Maasland, Holland) cultivator immediately after application. Wildflowers were broadcast-seeded at $2 \mathrm{~g}$ pure live seed per plot. Irrigation was applied daily at $4 \mathrm{~mm} \cdot$ day $^{-1}$ for 6 weeks after seeding and $13 \mathrm{~mm} \cdot$ week $^{-1}$ thereafter.

In the Nebraska study, tall plains coreopsis (Coreopsis tinctoria Nutt. ) and cosmos (Cosmos bipinnatus Cav.) plants lodged after heavy rains on $15(33 \mathrm{~mm})$ and 19 July (60 $\mathrm{mm}$ ) in the herbicide-treated plots but not the control. The lodged plants continued to grow, but remained lodged throughout the growing season. Herbicide-treated Indian blanket (Gaillardia pulchella Foug.) plants had uneven growth and a 2-week bloom initiation delay when compared to the control. After bloom, flowering appeared equal for control and herbicide treatments. About $35 \%$ of all species were adversely affected by the herbicide treatment,

Received for publication 30 Oct. 1989. Published as Paper no. 9059 Journal Series, Agricultural Research Division, Univ. of Nebraska, Lincoln, NE 68583. The cost of publishing this paper was defrayed in part by the payment of page charges. Under postal regulations, this paper therefore must be hereby marked advertisement solely to indicate this fact.

'Graduate Student, Dept. of Horticulture. ${ }^{2}$ Plant Breeder, Pure Seed Testing, Hubbard, Ore. ${ }^{3}$ Professor and Head, Dept. of Agronomy. ${ }^{4}$ Professor, Dept. of Horticulture. To whom reprint requests should be addressed.
Table 1. Stand ratings of annual and perennial wildflower species treated before establishment with EPTC/trifluralin (En) in studies conducted at Mead, Neb., and Hubbard, Ore.

\begin{tabular}{|c|c|c|c|c|}
\hline \multirow[b]{2}{*}{ Species } & \multicolumn{2}{|c|}{ Nebraska } & \multicolumn{2}{|c|}{ Oregon } \\
\hline & Control & $\mathrm{E} / \mathrm{T}$ & Control & $\mathrm{E} / \mathrm{T}$ \\
\hline & \multicolumn{4}{|c|}{ Annuals } \\
\hline Spurred snapdragon (Linaria maroccana Hook.f. ${ }^{2}$ ) & $8.5^{\mathrm{y}}$ & 7.5 & 9.0 & 9.0 \\
\hline Tall plains coreopsis (Coreopsis tinctoria Nutt.) & 8.0 & 8.0 & 9.0 & 8.5 \\
\hline California poppy (Eschscholzia californica Cham.) & 8.0 & 6.5 & 9.0 & 9.0 \\
\hline Baby's breath (Gypsophila elegans Bieb.) & 8.0 & 1.5 & 9.0 & 1.0 \\
\hline Garland chrysanthemum (Chrysanthemum coronarium L.) & 7.5 & $7.5^{-}$ & 9.0 & 9.0 \\
\hline Corn poppy (Papaver rhoeas L.) & 7.5 & 1.5 & 9.0 & 2.0 \\
\hline Mountain phlox [Linanthus grandiflonis (Benth.) Greene] & 7.5 & 1.0 & 7.0 & 5.0 \\
\hline Cosmos (Cosmos bipinnatus Cav.) & 7.5 & 6.5 & 4.5 & 4.0 \\
\hline Starflower (Scabiosa stellata L.) & 7.0 & 7.5 & ... & ..- \\
\hline Dwarf comflower (Centaurea cyanus L.) & 7.0 & 5.5 & 8.5 & 9.0 \\
\hline African daisy (Dimorphotheca sinuata DC.) & 7.0 & 8.0 & 8.0 & 7.0 \\
\hline Globe gilia (Gilia capitata Sims) & 7.0 & 1.5 & 8.0 & 1.0 \\
\hline Scarlet flax (Linum grandiflorum Desf. 'Rubrum') & 6.5 & 6.5 & 9.0 & 9.0 \\
\hline Birds eyes (Gilia tricolor Benth.) & 6.5 & 1.0 & 8.0 & 2.0 \\
\hline Annual Indian blanket (Gaillardia pulchella Foug.) & 6.5 & 5.5 & 5.5 & 5.0 \\
\hline Tall godetia [Clarkia amoena (Lehm.) A. Nels. \& Macbr.] & 5.5 & 3.0 & 9.0 & 9.0 \\
\hline Catchfly (Silene armeria L.) & 5.0 & 1.0 & 7.5 & 4.5 \\
\hline Mountain garland (Clarkia unguiculata Lindl.) & 5.0 & 4.0 & 7.5 & 6.5 \\
\hline Blue bells (Phacelia campanularia A. Gray.) - & 5.0 & 1.5 & 6.5 & 3.5 \\
\hline Tidy tips [Layia platyglossa (Fisch. \& C.A. Mey.)A. Gray] & 5.0 & 5.5 & 9.0 & 8.0 \\
\hline Rocket larkspur [Consoldia orientalis (J. Gay) Schrödinger] & 3.0 & 4.0 & 8.5 & 4.5 \\
\hline Baby blue eyes (Nemophila menziesii Hook. \& Arn.) & 2.0 & 2.0 & 9.0 & 5.0 \\
\hline Sweet alyssum [Lobularia maritima (L.) Desv.] & 1.0 & 1.0 & 8.0 & 5.0 \\
\hline \multirow[t]{2}{*}{ LSD $5 \%$} & \multicolumn{2}{|c|}{2.2} & \multicolumn{2}{|l|}{2.4} \\
\hline & \multicolumn{4}{|c|}{ Perennials } \\
\hline Black-eyed susan (Rudbeckia hirta L.) & $9.0^{y}$ & 9.0 & 9.0 & 8.5 \\
\hline Tall evening primrose (Oenothera erythrosepala Borb.) & 8.5 & 8.5 & -.. & -.- \\
\hline Creeping zinnia (Sanvitalia procumbens Lam.) & 8.5 & 7.5 & ... & -.- \\
\hline $\begin{array}{l}\text { Prairie coneflower [Ratibida columnifera (Nutt.) Woot. \& } \\
\text { Standl.] }\end{array}$ & 8.0 & 7.0 & 8.0 & 7.0 \\
\hline White yarrow (Achillea millefolium L.) & 8.0 & 8.0 & 9.0 & 8.0 \\
\hline Wild thyme (Thymus serpyllum L.) & 8.0 & 2.5 & 8.5 & 2.0 \\
\hline Roman chamonile [Chamaemelum nobile (L.) All.] & 8.0 & 8.0 & .... & -.. \\
\hline Dames rocket (Hesperis matronalis L.) & 7.5 & 6.5 & 9.0 & 8.5 \\
\hline Blue flax [Linum perenne subs. Lewisii (Pursh) Hult] & 7.5 & 7.0 & 8.0 & 8.5 \\
\hline Johnny jump-up (Viola cormuta L.) & 7.0 & 7.0 & 8.5 & 8.0 \\
\hline Red yarrow (Achillea millefolium L. 'Rubra') & 6.0 & 7.5 & 9.0 & 9.0 \\
\hline Maiden pinks (Dianthus deltoides L.) & 6.0 & 1.0 & 8.0 & 2.0 \\
\hline Dwarf lance-leaved corcopsis (Coreopsis lanceolata L.) & 6.0 & 6.0 & 3.5 & 8.0 \\
\hline Dwarf columbine (Aquilegia vulgaris L.) & 5.5 & 2.5 & 2.5 & 2.5 \\
\hline Siberian wallflower (Erysimum hieraciifolium L.) & 5.5 & 6.5 & 6.0 & 5.0 \\
\hline Small burnet (Poterium sanguisorba L.) & 5.5 & 6.5 & $\ldots$ & $\cdots$ \\
\hline Forget-me-not (Myosotis sylvatica Hoffm.) & 5.0 & 1.0 & 9.0 & 4.5 \\
\hline Purple coneflower [Echinacea purpurea (L.) Moench] & 4.5 & 4.0 & 7.5 & 3.5 \\
\hline Rocky mountain penstemon (Penstemon strictus Benth.) & 4.5 & 1.0 & 9.0 & 5.0 \\
\hline Sweet williams (Dianthus barbatus L.) & 4.0 & 1.0 & 7.5 & 4.0 \\
\hline Missouri primrose (Oenothera missouriensis Sims) & 2.0 & 2.5 & 6.5 & 7.5 \\
\hline Snow-in-summer (Cerastium biebersteinii DC.) & 2.0 & 1.0 & 9.0 & 6.0 \\
\hline English wallflower (Cheiranthus cheiri L.) & 1.5 & 2.0 & 8.0 & 9.0 \\
\hline LSD 5\% & \multicolumn{2}{|l|}{2.8} & \multicolumn{2}{|l|}{2.8} \\
\hline
\end{tabular}

${ }^{2}$ Hortus Third. 1976. Macmillan, New York.

'Stand values were based on a 1 to 9 rating scale, with $1=1 \%$ to $10 \%$ and $9=90 \%$ to $100 \%$ stand.

Values are means of two replications per treatment. 